\title{
Zur Methodik der Aktivitätsbestimmung von Membranenzymen (Acyltransferase und Lysophospholipase) $)^{1}$
}

\author{
Von E. Ferber, P. G. Munder und H. Fischer \\ Aus dem Max-Planik-Institut für Immunbiologie, Freiburg/Br.
}

(Eingegangen am 18. März 1966)

\begin{abstract}
Es wird eine Methode zur direkten Messung von ${ }^{14} \mathrm{C}$-markierten, auf Kieselgelpapier getrennten Phospholipiden und Fettsäuren im Flüssigkeitsscintillationszähler beschrieben. Der Umsatz der Membranenzyme Acyltransferase und Lysophospholipase kann mit dieser Methode ermittelt werden. Die Bestimmung der Aktivität dieser Enzyme erfolgt mittels ${ }^{14} \mathrm{C}$-markiertem Lysolecithin und ${ }^{14} \mathrm{C}$-markierten Fettsäuren. Fehlerquellen beim Arbeiten mit Lysophosphatiden, wie die Adsorption an Glasoberflächen und die Spontanhydrolyse im neutralen Milieu treten besonders unterhalb kritischer Konzentrationen auf. Es werden als Anwendungsbeispiele 1. die Erniedrigung der LL-acylierenden Reaktion in Antikörper-belegten Erythrocyten und 2. die Aktivierung der Lysophospholipase durch Dipyridamol behandelt.
\end{abstract}

A method is described for the direct measurement of ${ }^{14} \mathrm{C}$-phospholipids and ${ }^{14} \mathrm{C}$-fatty acids by liquid scintillation counting after their separation on paper impregnated with silicic acid. The activity of the two membrane enzymes, acyltransferase and lysophospholipase, can be determined with this method. The adsorption of lysophosphatides onto glass surfaces and their spontaneous hydrolysis in neutral buffers are sources of error, especially below certain critical concentrations. The application of the method is demonstrated 1. in the partial inhibition of the acyl transferase reaction in sheep erythrocytes coated with anti-Forssmann antibody and 2 . in the activation of lysophospholipase by dipyridamol (,,Persantin“).

Membranenzyme, die Lysophosphatide umsetzen, sind in letzter Zeit von mehreren Arbeitsgruppen untersucht worden und gewinnen an allgemein-biologischem und klinischem Interesse.

Nach Verfütterung markierter langkettiger Fettsäuren fanden van DEENEN und Mitarbeiter (1), daß reife Erythrocyten diese besonders in Lecithin, zum kleinen Teil auch in Kephalin, einbauen. Als Acceptoren dienen Lysophosphatide, insbesondere Lysolecithin $(2,3)$. Unsere Arbeitsgruppe stellte beim Studium der Hämolyse durch $\mathrm{LL}^{2}$ ) fest, daß diese bei kleinen, eben lytischen Konzentrationen von LL von der Aktivität bestimmter Membranenzyme abhängt (4). - Zwei enzymatische Reaktionen sind es, durch die in menschlichen Erythrocyten LL umgesetzt wird:

$$
\begin{aligned}
& \text { (1) Lysolecithin + Fettsäure } \frac{\text { Acyltransferasesystem }}{\mathrm{CoA}, \mathrm{ATP}, \mathrm{MB}}+\text { Lecithin } \\
& \text { (2) Lysolecithin } \stackrel{\text { Lysophospholipase }}{\longrightarrow} \text { GPC + Fettsäure }
\end{aligned}
$$

Die acylierende Reaktion (1) wurde von Landis und Merke (5) in Lebermikrosomen und von Oliveira und VAUGHAN (6) in Erythrocytenstroma gefunden. HEEMSKERK und VAN DEENEN (7) fanden als erste in Kaninchenerythrocyten die Lysophospholipasereaktion (2), durch die LL zu Glycerylphosphorylcholin und Fettsäure abgebaut wird.

Da feststeht, daß die genannten Enzyme nicht auf Erythrocyten bescbränkt sind, sondern eine weite Verbreitung haben, und da ferner Anhaltspunkte dafür vorliegen, da $B$ durch bestimmte Pharmaka, u. a. durch solche, die Transportvorgänge beeinflussen, die Aktivi-

1) Die Triviailnamen Acyltransferase und Lysophospholipase werden hier gebraucht für die Enzyme: Acyl-CoA: LysolecithinAcyltransferase und Lysolecithin-Acylhydrolase EC 3115.

2) Abkïrzungen: LI = Lysolecithin (Lysophosphatidylcholin); Lec = Lecithin (Phosphatidylcholin); Sph = Sphingomyelin; Keph $=$ Colamin- Kephalin (Phosphatidylaethanolamin); GPC = Glycerylphosphorylcholin. tät dieser Enzyme verändert wird (8), sollen hier einige methodische Voraussetzungen für die Messung dieser Reaktionen kurz geschildert werden.

\section{Methodik}

Die Aktivität der Acyltransferase und der Lysophospholipase wurde mit Hilfe markierter Substrate bestimmt. Als markiertes Substrat für die Acyltransferasereaktion verwendeten wir $\left[{ }^{14} \mathrm{C}\right]-\mathrm{O} l-$ säure und $\left.\left[{ }^{14} \mathrm{C}\right]-P a l m i t i n s a ̈ u r e^{3}\right)$ und zur Messung der Aktivität der Lysophospholipase [1-Acyl-14 C]-Lysolecithin (Darstellung s. (4)). Die Lipidextrakte der Reaktionsansätze wurden auf Kieselgel-imprägniertem Papier') nach MaRINETTI (9) getrennt und mit Rhodamin $6 G$ gefärbt. Die Abbildung 1 zeigt schematisch eine solche Trennung.

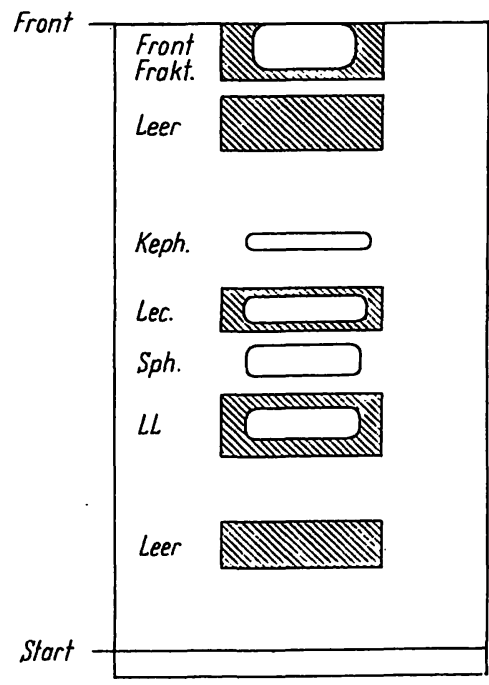

Abb. 1

Schema eines Lipidchromatogramms.

Fließmittel: Diisobutylketon/Essigsäure/Wasser 8:5:1 ( Laufzeit: $3 \mathrm{Stdn} . / 25^{\circ}$; Frontfrakt. = Fettsäuren, Cholesterin, Cholesterinester und Neutralfette

3) Radiochemical Center Amersham, England.

4) Kieselgelpapier Nr. 289, Fa. Schleicher \& Schüll, Dassel 
$\mathrm{Da}$ die Aktivität solcher getrennten Fraktionen sehr gering war, konnten die Chromatogramme nicht kontinuierlich mit Hilfe eines Radiopapierchromatographen ausgemessen werden. Sie läßt sich jedoch im Liquid-Scintillationsspektrometer ${ }^{1}$ ) bestimmen. Hierzu wurden die in Abbildung 1 schraffiert dargestellten Zonen (LL, Lec, Frontfraktion und ein oder zwei Papierleerwerte) in 1,5x $3,5 \mathrm{~cm}$ breite Streifen zerschnitten, diagonal in normale Meßgläser gebracht und mit $16 \mathrm{~m} /$ Scintillator $(5 \mathrm{~g}$ 2,5-Diphenyloxazol $+0,3 \mathrm{~g}$ 1,4-bis-2(4-Methyl-5-phenyloxazolyl)-benzol in $1000 \mathrm{~m} /$ Toluol) im Scintillationsspektrometer gemessen.

Die Gewinnung der Hämolysate und Stromapräparationen ebenso wie die Methoden der Lipidextraktion und Chromatographie wurden an anderer Stelle ausführlich beschrieben (4).

\section{Ergebnisse und Diskussion}

\section{Vermeidung von Zäblverlusten durch Quencheffekt}

Die Messung im Flüssigkeitsscintillationszähler setzt peinliche Berücksichtigung von Zählverlusten durch Quench voraus. Die sicherste Methode zur Erfassung solcher Verluste ist die Verwendung eines inneren Standards. Wird die Radioaktivität jedoch in einer inhomogenen Probe (wie hier Papierzone im Scintillator) bestimmt, so läßt sich dieses Verfahren nicht anwenden. Für wasserlösliche ${ }^{14} \mathrm{C}$ - und ${ }^{3} \mathrm{H}$-markierte, auf Filterpapier aufgetragene Substanzen sind zwar Methoden zur direkten Messung im Scintillationszähler beschrieben (10-14), das Verhalten markierter Phospholipide mußte jedoch zunächst in Vorversuchen geprüft werden. Dabei stellte sich heraus, daß bei der Messung ungefärbter Zonen kein Zählverlust gegenüber der idealen Lösung im Scintillator selbst auftritt (Wirkungsgrad 83\%).

Wie Tabelle 1 zeigt, tritt jedoch nach Anfärbung mit Rhodamin bei allen analysierten Lipidfraktionen ein erheblicher Quencheffekt ein. (Die Aktivität der nicht gefärbten Fraktionen wurde $=100 \%$ gesetzt.)

Tab. 1

Quench durch Anfärben mit Rhodamin 6G. (Aktivität der nicht gefärbten Fraktionen $=100 \%$ )

\begin{tabular}{lcccc}
\hline Auftragsmenge & \multicolumn{2}{c}{$5 \mathrm{~m} \mu \mathrm{Mol} / 2 \mathrm{~cm}$} & \multicolumn{2}{c}{$105 \mathrm{~m} \mu \mathrm{Mol} / 2 \mathrm{~cm}$} \\
\hline $\begin{array}{l}\text { nicht } \\
\text { gefärbt }\end{array}$ & $\begin{array}{c}\text { nach } \\
\text { Rhodamin- } \\
\text { färbung }\end{array}$ & $\begin{array}{c}\text { nicht } \\
\text { gefärbt }\end{array}$ & $\begin{array}{c}\text { nach } \\
\text { Rhodamin- } \\
\text { färbung }\end{array}$ \\
\hline $\begin{array}{l}\text { Lysolecithin } \\
\text { Lecithin }\end{array}$ & 100 & 79 & 100 & 95 \\
Ölsäure & 100 & 78 & 100 & 94 \\
& 100 & 50 & 100 & 66 \\
\hline
\end{tabular}

Verwendete Lipide: [1-Acyl-14-C]-Lysolecithin und [1,2-Acyl$\left.{ }^{14} \mathrm{C}\right]$-Lecithin aus Leber nach (4) und [1$\left.{ }^{14} \mathrm{C}\right]$-Ölsäure.

Für LL und Lec läßt sich dieser Quencheffekt durch zusätzlichen Auftrag von nịcht markierten Lipiden ausgleichen, nicht jedoch der Zählverlust der Ölsäure. Er schwankt bei geringen Änderungen der Fettsäuremenge und der Anfärbung und läßt sich daher nicht durch eine einfache, rechnerische Korrektur ausgleichen. Werden jedoch gemeinsam mit $\left[{ }^{14} \mathrm{C}\right]$-Ölsäure Erythrocytenlipide chromatographiert, die ebenfalls zur Front wandern

1) Tricarb Serie 3000, Fa. Packard Instr. Co., La Grange, Ill. USA.
(Cholesterin, Cholesterinester, Neutralfette und Fettsäuren), und $z$ war in Mengen, die aliquoten Teilen von $0,05-0,1 \mathrm{ml}$ Sediment $/ 2 \mathrm{~cm}$ entsprechen, so ist $\mathrm{der}$ Quench völlig aufgehoben (Tab. 2).

Tab. 2

Quench-Verhalten der Olsäure.

(Aktivität der nicht gefärbten Fraktion $=100 \%$ )

\begin{tabular}{lcc}
\hline & $\begin{array}{c}{\left[1-{ }^{14} \mathrm{C}\right]-O ̈ l s a ̈ u r e} \\
1 \mathrm{~m} \mu \mathrm{Mol} / 2 \mathrm{~cm}\end{array}$ & $\begin{array}{c}{\left[1-{ }^{14} \mathrm{C}\right]-O ̈ l s a ̈ u r e} \\
1 \mathrm{~m} \mu \mathrm{Mol} / 2 \mathrm{~cm} \\
+ \text { Frontfraktion }\end{array}$ \\
\hline $\begin{array}{l}\text { nicht gefärbt } \\
\text { nach Rhod- } \\
\text { aminfärbung }\end{array}$ & 100 & 100 \\
\hline
\end{tabular}

Frontfraktion $=$ Lipidgemisch (s. Abb. 1), aliquoter Teil von $0,05 \mathrm{~m} l$ Erythrocytensediment.

Cholesterin allein hebt den Zählverlust nicht auf, so daß entweder eine der anderen Komponenten oder ihre Kombination für diesen Effekt verantwortlich zu machen sind.

Sorgt man dafür, daß nicht kleinere Gesamtmengen an LL und Lec als $100 \mathrm{~m} \mu \mathrm{Mol}$ (etwa 50-70 $\mu \mathrm{g}$ ) und nicht weniger als $0,05 \mathrm{ml}$ Erythrocytensediment bzw. entsprechende Lipidmengen eingesetżt werden, so kann der kleine noch vorhandene Quencheffekt (etwa 2-6\%) vernachlässigt werden.

\section{Spontane Hydrolyse von LL}

In Chloroform/Methanol bei $+4^{\circ}$ und aùch in wäßrigen Lösungen bei $-70^{\circ}$ ist LL über mehrere Monate stabil. Bei $37^{\circ}$ jedoch wird in wäßriger Lösung bei pH 7 ein Teil der Fettsäure abgespalten. Wie Abbildung 2 zeigt, hängt das Ausmaß der Hydrolyse stark von der Konzentration von LL āb. So sind bei einer Konzentration von $10 \mathrm{~m} \mu \mathrm{Mol} / \mathrm{m} l(=5 \mu \mathrm{g} / \mathrm{m} l)$ nach $3 \mathrm{Stdn} .30 \%$ des LL gespalten, während bei $50 \mathrm{~m} \mu \mathrm{Mol} / \mathrm{m} l$ nur $12 \%$ hydrolysiert sind.

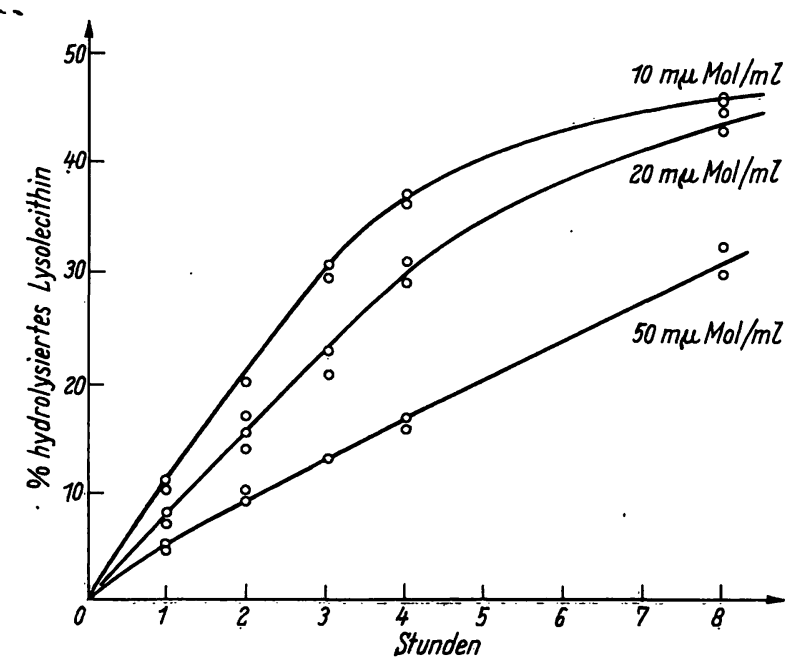

Abb. 2

Spontane Hydrolyse von Lysolecithin.

Reaktionsbedingungen: In $1 \mathrm{~m} l 0,1 \mathrm{M}$ Phosphatpuffer $\mathrm{pH} 7,4$ waren $10-50 \mathrm{~m} \mu \mathrm{Mol}$ [1-Acyl-14 C]-LL gelöst; Inkubation bei $37^{\circ}$; anschließend Extraktion und Chromatographie wie beschrieben. Hydrolyserate in $\%=\frac{\text { Aktivität der freigesetzten Fettsäure }}{\text { Gesamtaktivität }} \times 100$ 
Dieses Verhalten beruht wahrscheinlich auf der Bildung von Micellen. Die Hydrolyse kommt zum Stillstand (teils in der Abbildung nicht dargestellt), wenn etwa 50\% des LL gespalten sind. Die Ursache hierfür ist noch nicht bekannt. Da es bei unseren Reaktionsbedingungen jedoch unwahrscheinlich ist, daß eine meßbare Rückreaktion stattfindet, prüfen wir z. Z. die Frage, ob evtl. die Spaltprodukte (Fettsäure und GPC) einen stabilisierenden Effekt auf LL ausüben.

\section{Adsorption von $L L$ an Glasoberflächen}

Beim Arbeiten mit wäßrigen Lösungen von Lysophosphatiden in geringen Konzentrationen ist neben der Spontanhydrolyse die Adsorption an Glasoberflächen als mögliche Fehlerquelle zu beachten. Wie Abbildung 3 zeigt, werden bei Konzentrationen von $20 \mathrm{~m} \mu \mathrm{Mol} / \mathrm{m} l$ $(=10 \mu \mathrm{g} / \mathrm{m} l) 50 \%$ des LL an der Glaswand adsorbiert. Unter unseren Versuchsbedingungen tritt eine Sättigung erst bei etwa $100 \mathrm{~m} \mu \mathrm{Mol} / \mathrm{m} l$ ein. Enthält der Ansatz jedoch Erythrocyten (10-proz. Suspension), so werden bei Verwendung der gleichen Konzentrationen etwa $95 \%$ des eingesetzten LL an die Erythrocyten gebunden; die restlichen $5 \%$ verteilen sich auf freies und an Glas adsorbiertes LL.

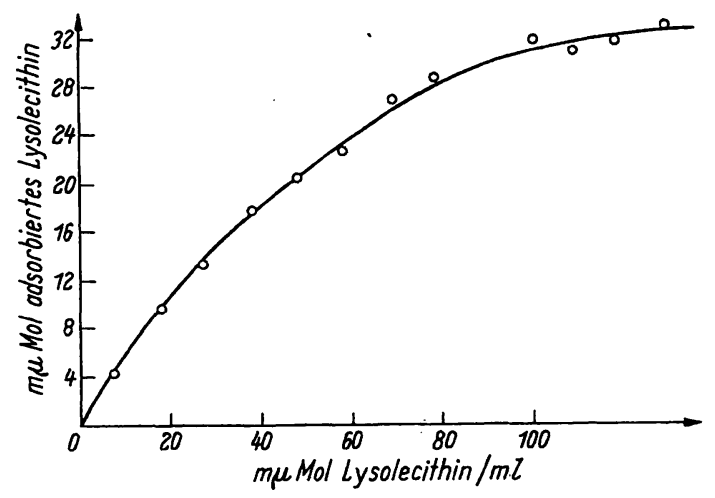

Abb. 3

Adsorption von Lysolecithin an Glasoberflächen.

Jeder Ansatz enthielt in $1 \mathrm{~m} l 0,1 \mathrm{M}$ Phosphatpuffer $\mathrm{pH} 7,4$ 7-107 m $\mu \mathrm{Mol}$ [1-Acyl- ${ }^{14} \mathrm{C}$ ]-LL; Inkubation $5 \mathrm{Min} . / 25^{\circ}$; $\varnothing$ der Reagenzgläser $=17 \mathrm{~mm}$

\section{Enzymaktivität in Vollbämolysat und Stroma}

Acyltransferase und Lysophospholipase sind stromagebunden (4) und werden am besten im Hämolysat bestimmt. Die größten Enzymaktivitäten werden in „Vollhämolysat" gemessen, in dem beide Reaktionen etwa $10 \mathrm{mal}$ schneller verlaufen als in intakten Erythrocyten. Dialysiert man „Vollhämolysat", das durch dreimaliges Gefrieren und Tauen von Erythroçytensediment hergestellt wurde, 24 Stdn. bei $4^{\circ}$ gegen 0,02 M Phosphatpuffer $\mathrm{pH} 7,4$, so wird, wie Tabelle 3 zeigt, besonders stark die Lysophospholipase inaktiviert. In isolierten Stromapräparationen ist die Aktivität der Enzyme, wie früher gezeigt wurde (4), um so geringer, je häufiger sie gewaschen wurden. Aber auch die Methode des Zellaufschlusses (Gefrierhämolyse, osmotische Hämolyse) hat starken Einfluß auf die Aktivität der Enzyme (Tab. 3).
Tab. 3

Enzymaktivität in verschiedenen Membranpräparationen menschlicher Erythrocyten.

Werte in $\mathrm{m} \mu \mathrm{Mol}$ umgesetzten $\mathrm{LL} / \mathrm{m} /$ Ery.-Sed. $\mathrm{x}$ Min.

\begin{tabular}{lccc}
\hline & $\begin{array}{c}\text { Lysophospho- } \\
\text { lipase }\end{array}$ & $\begin{array}{c}\text { Acyltrans- } \\
\text { ferase }\end{array}$ \\
\hline Gefrier- & dialysiert & 5,0 & 2,4 \\
Vollhämolysat & nicht dialysiert & 44,0 & 4,0 \\
Gefrierstroma & 2,0 & 3,0 \\
Osmotisches & 1,0 & 1,0 \\
\hline Stroma & & 1,0 & \\
\hline
\end{tabular}

Jeder Reaktionsansatz enthielt in einem Endvolumen ron $1,0 \mathrm{~m} /$ : $55 \mathrm{~m} \mu \mathrm{Mol}\left[1-\right.$ Acyl $\left.^{-14} \mathrm{C}\right]-\mathrm{LL} ; 40 \mathrm{~m} \mu \mathrm{Mol}$ Olsäure; $56 \mathrm{~m} \mu \mathrm{Mol} \mathrm{CoA}$; $10 \mu \mathrm{Mol} \mathrm{ATP;} 10 \mu \mathrm{Mol} \mathrm{IgCl}_{2} ; 65 \mu \mathrm{Mol}$ Phosphatpuffer $\mathrm{pH} 7,4$; $0,1 \mathrm{ml}$ Hämolysat = aliquot von $0,01 \mathrm{ml}$ Ery.-Sed.; Inkubation $30 \mathrm{Min} \cdot / 37^{\circ}$.

Beide Präparationen wurden so gewonnen, daß ein Teil Erythrocytensediment mit 10 Teilen Wasser gemischt, das Stroma durch $30 \mathrm{Min}$. Zentrifugieren bei $34800 \mathrm{~g}$ gewonnen und im Ausgangsvolumen mit $0,1 \mathrm{M}$ Phosphatpuffer resuspendiert wurde. Der einzige Unterschied beider Präparate bestand darin, daß bei der Gewinnung von „Gefrierstroma" zu Beginn die Erythrocyten durch 3 maliges Gefrieren und Tauen hämolysiert wurden, während beim osmotischen Stroma die Hämolyse lediglich durch destilliertes W:asser erfolgte. Wie die Tabelle 3 zeigt, findet man im "Gefrierstroma", das mikroskopisch aus stärker rupturierten Zellhüllen besteht, eine höhere Aktivität der Lysophospholipase und der Acyltransferase als im „osmotischen" Stroma.

Anwendungsbeispiele

Aktivierung der Lysophospholipase (Reaktion (2)) durch Pharmaka

An anderer Stelle (8) wurde kurz mitgeteilt, daß Dipyridamol („Persantin") die Lysophospholipase von Erythrocyten aktiviert. Wir wählten Persantin, da seine permeabilitätsändernden Wirkungen durch die Untersuchungen von Gerlach und Deutrcke $(15,16)$ eingehend beschrieben wurden. An dieser Stelle soll ein solcher Versuch als Beispiel für die Bestimmung der Aktivität der Membranenzyme dargestellt werden. Tabelle 4 gibt die Anfangsgeschwindigkeiten der Re-

Tab. 4

Wirkung von Dipyridamol auf die Aktivität der Lysophospholipase Werte in $\mathrm{m} \mu \mathrm{Mol}$ umgesetzten $\mathrm{LL} / \mathrm{m} /$ Ery.-Sed.

\begin{tabular}{cccc}
\hline $\begin{array}{c}\text { Inkub.-dauer } \\
\text { (Min.) }\end{array}$ & Kontrolle & $\begin{array}{c}\text { Dipyridamol } \\
(0,1 \mu \mathrm{Mol} / \mathrm{m} l)\end{array}$ & $\begin{array}{c}\% \text { Aktivierung } \\
\text { (Kontrolle } \\
=100 \%)\end{array}$ \\
\hline 10 & 144 & 408 & 283 \\
20 & 272 & 678 & 249
\end{tabular}

Der Reaktionsansatz enthielt in einem Endvolumen von $1,0 \mathrm{~m} /$ :

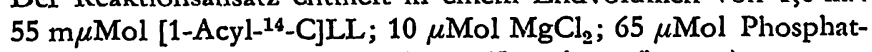
puffer $\mathrm{pH} 7,4 ; 0,025 \mathrm{~m} l$ dialysiertes Hämolysat (human). 
aktion (2) in menschlichem Hämolysat wieder. Unter Dipyridamol ist sofort - ohne Vorinkubation - die Reaktionsgeschwindigkeit fast $3 \mathrm{mal}$ so hoch wie in der Kontrolle.
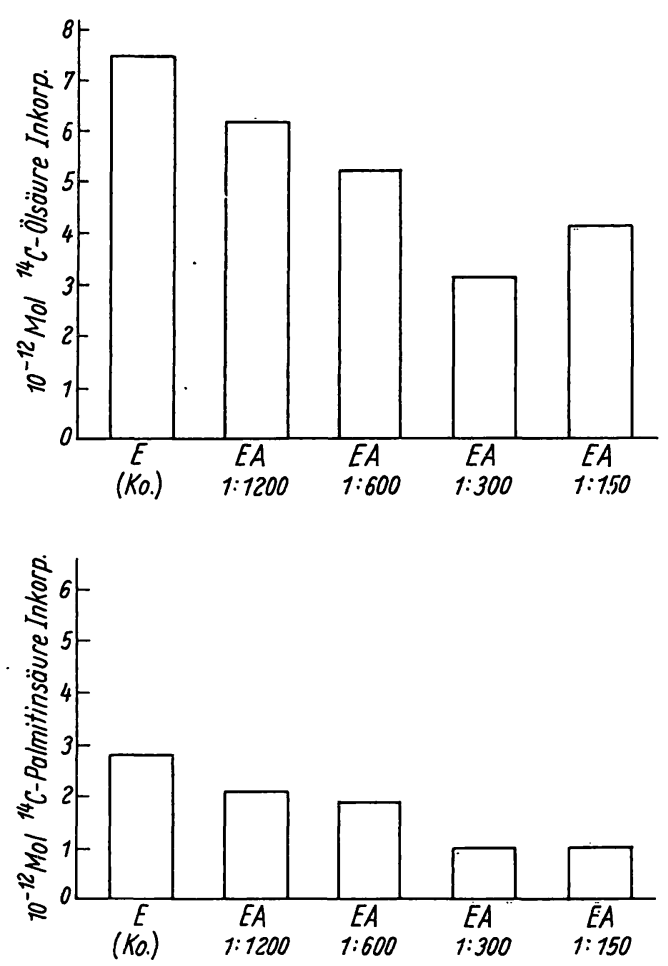

Abb. 4 und 5

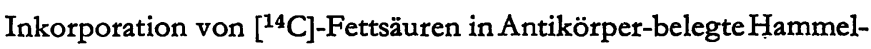
erythrocyten (EA-Zellen).

Herstellung Antikörper-belegter Hammelerythrocyten: $30 \mathrm{ml}$ 15-proz. Hammelerythrocytensuspension wurden mit $30 \mathrm{ml}$ Amboceptorverdünnung $(1: 150$ bis $1: 1200) 30 \mathrm{Min} . / 37^{\circ}$ inkubiert. Nach der Inkubation $3 \mathrm{mal}$ Waschen der Zellen mit phys. Kochsalzlösung. Reaktionsansatz: Jeder Ansatz enthielt in einem Endvol. von $1,0 \mathrm{ml}$ (Pillemer-Puffer): $0,5 \mathrm{~m} \mu \mathrm{Mol}\left[{ }^{14}{ }^{14} \mathrm{C}\right]$-Ölsäure oder $\left[1{ }^{14} \mathrm{C}\right]$-Palmitinsäure; $2,5 \mathrm{~m} \mu \mathrm{Mol} \mathrm{LL} ; 0,1 \mathrm{ml}$ Sediment; Inkubation $120 \mathrm{Min} . / 37^{\circ}$
Aus noch nicht abgeschlossenen Versuchen geht hervor, $\mathrm{da}$ B eine ähnliche Beschleunigung des Lysolecithinabbaus durch einige antiphlogistisch wirkende Substanzen hervorgerufen wird (17).

\section{Aktivität der Acyltransferase (Reaktion (1)) in Antikörper- beladenen Zellen}

Werden Hammelerythrocyten mit käuflichem Amboceptor $^{1}$ ) (Anti-Forssmann-Antikörper) in Verdünnungen von 1:150 bis 1:1200 sensibilisiert, so sind sie gegenüber LL empfindlicher als nicht sensibilisierte Zellen (4). Um zu prüfen, ob diese Ĺabilisierung durch eine Hemmung der Acyltransferase oder der Lysophospholipase hervorgerufen wird, bestimmten wir die Aktivität beider Enzyme in intakten Erythrocyten.

Die Messung der Reaktion (2) mit Hilfe von ${ }^{14} \mathrm{C}$-LL zeigte, daß die Lysophospholipaseaktivität nicht verändert ist. Die Acyltransferasereaktion (1) ist bei dieser Versuchsanordnung (mit markiertem LL) ebenfalls nicht verlangsamt. Wurde dagegen die Aktivität der Acyltransferase mit $\left[{ }^{14} \mathrm{C}\right]$-Ölsäure oder $\left[{ }^{14} \mathrm{C}\right]$-Palmitinsäure gemessen, so fand sich, wie die Abbildungen 4 und 5 zeigen, eine Erniedrigung des Umsatzes, die deutlich von der Antikörperkonzentration abhing.

Warum diese Verlangsamung der Acylierung nur bei Verwendung markierter Fettsäuren gemessen wird, kann z. Z. noch nicht befriedigend erklärt werden. Diese Diskrepanz könnte dadurch bedingt sein, da $\beta$ in antikörperbelegten Zellen nur die Aktivierung der Fettsäuren mit CoA (Ligase-Reaktion) und nicht die Transferase-Reaktion langsamer verläuft. Dies wird z. Z. mit $\left[{ }^{14} \mathrm{C}\right]$-Acyl-CoA geprüft.

Wir danken den med.-technischen Assistentinnen Frl. INGRID AernNelt und Frl. Karin Zwetschke für wertvolle Mitarbeit. Die Arbeit wurde z. T. mit Mitteln der Research Corporation, New York, ausgeführt.

1) Fa. Behring-Werke, Marburg/L.

\section{Literatur}

1. Kögl, F., J. de Gịer, I. Mulder und L. L. M. van Deenen, Biochim. biophysica Acta (Amsterdam) 43, 95 (1960). - 2. vAN DeEnen, L. L. M., J. DE Gier, U. M. T. Houtsmuller, A. MontFORT und E. MULDER in A. C. Frazer: Biochemical Problems of Lipids, S. 404, Elsevier, Amsterdam (1963). - 3. MuLDER, E., J. De Grer und L. L. M. van Deenen, Biochem. biophysica Acta (Amsterdam) 70, 94 (1963). - 4. Munder, P. G., E. Ferber und H. FrsCHER, Z. Naturforsch. $20 b, 1048$ (1965). - 5. LANDs, W. E. M. und I. MerkL, J. biol. Chemistry 238, 898 (1963). - 6. OlIveira, M. M. und M. Vaughan, Federat. Proc. 21, 296 (1962). 7. Heemskerk, C. T. H. und L. L. M. van Deenen, Kon. Nederl. Acad. Wetensch. Amsterdam, Proc. S. B 67, 181 (1964). - 8. Ferber, E., P. G. Munder und H. Fischer, Biochem. Z. 344 ,
218 (1966) - 9. Marinettr, G. V., J. Erbland und J. Kochen, Federat. Proc. 16, 837 (1957). - 10. WANG, C. H. und D. E. JonES, Biochem. biophysic. Res. Commun. 1, 203 (1959). - 11. GEIGER, J. W. und L. B. WRIGHT, Biochem. biophysic. Res. Commun. 2, 282 (1960). - 12. Bousquet, W. F. und J. E. Christian, Analytic Chem. 32, 722 (1960). - 13. Lofrfieid, R. B., Atomlight No. 13 (1960). - 14. Davidoson, E. A., Packard Technical Bull. No. 4 (1962). - 15. Gerdach, E., B. Deutrcke und J. Duhm, Pflügets Arch. ges. Physiol. Menschen Tiere 280, 243 (1964). - 16. Devticke, B. J., Duhm und E. Gerrach, Pflügers Arch. ges. Physiol. Menschen Tiere 280, 275 (1964). - 17. Ferber, E., P. G. Munder und H. Fischer, in Vorbereitung.

Professor Dr. H. Fischer

Max-Planck-Institut für Immunbiologie 78 Freiburg/Br.-Zähringen Stübeweg 